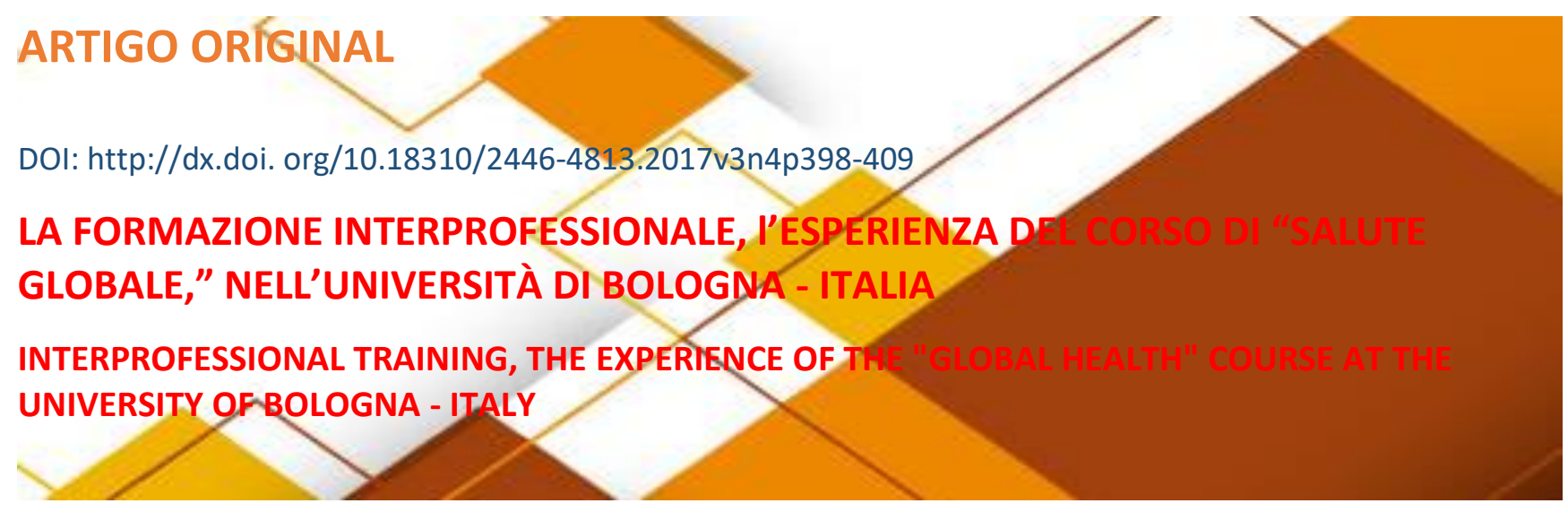

\title{
ARDIGÓ MARTINO
}

Medico, specialista in salute pubblica, phd in cooperazione internazionale allo sviluppo presso I'Università di Bologna, Ricercatore a Tempo Determinato presso L'Università di Bologna.

E-mail: ardigo.martino@gmail.com

\section{ASTRATTO}

Obiettivo: obiettivo della ricerca è valutare gli effetti della formazione interprofessionale ed in comunità attraverso le percezioni degli studenti, dei docenti e dei tutor. Metodologia: la metodologia utilizzata è qualitativa, basata sulla ricerca-formazione-intervento. Sono stati utilizzati come materiali le valutazioni degli studenti, i diari di campo di studenti, tutor e docenti, le informazioni raccolte attraverso un focus group finale a cui hanno partecipano docenti, tutor e studenti volontari. I materiali sono stati analizzati attraverso l'analisi di contenuto e triangolati con le informazioni provenienti dalla letteratura. Risultati: la ricerca ha mostrato come la formazione interprofessionale rappresenti una sfida per docenti, tutor e studenti, perché necessita dello sviluppo di metaconoscenze e metacompetenze non appartenenti a nessun campo disciplinare, ma generate dal lavoro in equipe applicato a condizioni concrete del quotidiano. L'apprendimento di tali competenze è gravoso per gli studenti, perché mette in gioco conoscenze e modalità di lavoro anche molto differenti da quelle a cui sono abituati nella formazione disciplinare. D'altra parte, devono essere attentamente studiati e tenuti sotto controllo i fattori strutturali prossimi e distali che interferiscono con la formazione, a partire dalle relazioni tra professioni ed al curriculum occulto dei corsi di laurea. Conclusioni: l'impegno per lo sviluppo della formazione interprofessionale dovrebbe avvenire a livello di università e non di facoltà, coinvolgere la comunità con l'obiettivo di superare le barriere disciplinari e facilitare l'interazione degli studenti con i problemi complessi della comunità. La formazione interprofessionale richiede tempo, pertanto deve essere incorporata nei curriculum obbligatori e con un numero di crediti adeguato.

PAROLE CHIAVE: Istruzione; Équipe d'assistenza ai pazienti cronici; Alleanza tra Università, servizio e comunità; Formazione professionale;

\section{ABSTRACT}

Objective: research objective is to evaluate the effects of community based inter-professional training through students, teachers and tutors perceptions. Methodology: we used a qualitative methodology, based on researchtraining-intervention framework. Materials used are students course evaluation, students', tutors' and lecturers' field notes, and information gathered through a final focus group. The materials were analyzed through content analysis and triangulated with literature findings.Results: research showed that interprofessional training represents a challenge for teachers, tutors and students. In 
fact, it requires the development of metaknowledge and meta-competences not belonging to any disciplinary field, but generated through community based teamwork. Developing those skills is toilsome for students because it brings into play knowledge and working methods different from those they are used in their disciplinary fields. Moreover, proximal and distal structural factors, such as power relations between professions and effects of hidden curriculum, are involved in inter-professional training. These factors should be carefully studied, monitored and amended. Conclusions: the commitment to the development of interprofessional training should take place at the university level with the aim of overcoming disciplinary barriers and should encourage students' interaction with the complex problems of the community. The interprofessional training requires time and resources, so it should be incorporated into official curriculum with an adequate amount of academic credits.

KEYWORDS: Education; Patient Assistance Team; Community-Institution Relations; Professional Training

RESUMO: Objetivo: o objetivo da pesquisa é avaliar os efeitos da formação interprofissional baseada na comunidade por meio de percepções de alunos, professores e tutores. Metodologia: utilizou-se uma metodologia qualitativa, baseada na estrutura de pesquisatreinamento-intervenção. Os materiais utilizados são a avaliação do curso dos alunos, notas de campo dos alunos, dos tutores e dos professores e as informações coletadas por meio de um grupo de foco final. Os materiais foram analisados por meio da análise de conteúdo e triangulados com os achados da literatura. Resultados: a pesquisa mostrou que o treinamento interprofissional representa um desafio para professores, tutores e estudantes. De fato, isso requer o desenvolvimento de meta-conhecimento e meta-competências não pertencentes a qualquer campo disciplinar, mas geradas por meio do trabalho em equipe baseado na comunidade. Desenvolver essas habilidades é trabalhoso para os estudantes, porque põe em jogo conhecimentos e métodos de trabalho diferentes daqueles que são usados em seus campos disciplinares. Além disso, fatores estruturais proximais e distais, como relações de poder entre profissões e efeitos do currículo oculto, estão envolvidos na formação interprofissional. Esses fatores devem ser cuidadosamente estudados, monitorados e alterados. Conclusões: o compromisso com o desenvolvimento da formação interprofissional deve ocorrer no nível universitário, com o objetivo de superar as barreiras disciplinares e estimular a interação dos estudantes com os complexos problemas da comunidade. A formação interprofissional requer tempo e recursos, por isso deve ser incorporada ao currículo oficial com uma quantidade adequada de créditos acadêmicos.

PALAVRAS-CHAVE: Educação; Equipe de Assistência ao Paciente; Relações ComunidadeInstituição; Capacitação Profissional.

\section{INTRODUZIONE}

Nel contesto italiano, il rapido invecchiamento della popolazione e la riduzione della natalità hanno generato una forte domanda assistenziale in relazione ai pazienti cronici, che, con una aspettativa di vita attuale superiore ai 90 anni, sono frequentemente affetti da pluripatologie. Nella Regione Emilia-romagna, nel Nord Italia (la stessa regione in cui è stata realizzata questa ricerca), circa una persona su 4 ha più di 65 anni di età, e quasi una su 10 ha più di 85 anni . Al di là di questo, il gruppo dei "giovani" della popolazione è costituito da una 
porzione consistente di migranti che nella regione sono intorno al $12 \%$ della popolazione totale. Questo scenario crea un contesto di grande complessità, dove i problemi di salute sono frequentemente associati a fattori sociali, economici, politici e culturali, allo stesso tempo radicati nei processi globali, ma con concreti effetti locali, rappresentando una sfida per l'intera comunità, non solo per i servizi sociali e sanitari. A partire da questi presupposti, in Emilia Romagna dall'anno 2010 si è cominciato ad implementare servizi di cure primarie sulla scia della normativa sulle "Case della Salute", ma in un contesto fortemente vincolato all'ospedale ed alle cure specialistiche, con un uso abbondante della tecnologia, un'organizzazione prevalentemente verticale, accompagnata da una formazione dei professionisti anch'essa prevalentemente ospedalocentrica e centrata sulla specialità. La riorganizzazione del sistema, pertanto, rappresenta una sfida culturale ma anche un possibile punto di svolta nella storia del sistema di salute italiano. II cambiamento culturale, a sua volta, è strettamente correlato alla capacità dei professionisti di riadattare l'assistenza al nuovo contesto e, come sostenuto dalla stessa Organizzazione Mondiale della Sanità (OMS), il mancato re-training ed il re-profiling dei professionisti sanitari in un'ottica di lavoro in equipe e reti inter-settoriali che mettano al centro il paziente è uno dei principali ostacoli alla transizione dei servizi sanitari. ${ }^{i}$ Per questo motivo, il Centro Studi e Ricerche in Salute Internazionale e Interculturale dell'Università di Bologna (CSI) ha definito un progetto di ricercaformazione-intervento, con la finalità di sperimentare ed analizzare possibili strategie di formazione e la loro applicabilità all'interno del contesto della Regione Emilia-Romagna.

\section{MATERIALI E METODI}

Nel corso degli anni accademici 2016 e 2017 è stato utilizzato come campo di sperimentazione il corso opzionale "Salute Globale, Determinanti Sociali, diseguaglianze in Salute e strategie di Primary Health care", offerto dal Corso di Laurea in Medicina e Chirurgia della UNIBO.

La metodologia di ricerca usata si ispira alla ricerca-intervento partecipata (PAR) arricchita della dimensione della formazione, costituendo così una forma originale di ricerca-formazioneintervento. ii,iii L'obiettivo del processo è produrre conoscenza a partire dalle esperienze degli attori coinvolti, conoscenza che poi possa essere utilizzata per l'empowerment degli 
attori stessi. ${ }^{\mathrm{iv}, v}$ Pertanto, la ricerca formazione intervento si qualifica per una forte associazione tra teorie e pratiche che autori come Heller riconoscono confluire nelle prassi. vi Inoltre, la metodologia ha l'obiettivo di approssimare università, servizi (sociali e sanitari) e comunità con l'intento di stimolare una formazione accademica basata sulle pratiche contestuali, facilitando lo sviluppo di competenze etiche, politiche e tecniche nei futuri professionisti così come nei formatori e nei ricercatori. (Figura 1)

La ricerca ha avuto uno scopo più ampio rispetto a quanto riportato nel presente articolo. Sono qui presentati gli elementi salienti emersi rispetto al tema della interdisciplinarità e della formazione interprofessionale. II Corso opzionale consta di 24 ore di attività, le attività in campo, però, possono prevedere un carico orario più ampio del pianificato. II Corso opera su differenti segmenti della tassonomia di Bloom, vii coinvolgendo gli studenti in attività in aula e nella comunità, ed è organizzato in 3 blocchi concettuali: una prima parte teorica, che affronta i temi della transizione epidemiologica e demografica e gli effetti della globalizzazione nei processi salute malattia a livello locale; una seconda parte del corso riguarda le metodologie e le tecniche di lavoro multiprofessionali ed intersettoriali, l'etica della ricerca intervento e la responsabilità sociale dell'incontro con la comunità; l'ultima parte è quella dell'immersione, dove un problema-dispositivo funziona come punto di partenza per le riflessioni rispetto alla responsabilità dei sistemi sanitari nel contrastare le diseguaglianze sociali ed i loro effetti in salute e a perseguire l'equità. viii

II corso è aperto, senza restrizioni, a tutti gli interessati e possono partecipare studenti di tutte le facoltà, di qualsiasi anno e di qualsiasi grado, professionisti e cittadini. II numero di partecipanti non è fisso ma dipende dalla capacità ricettiva del campo, ossia degli attori comunitari, delle loro organizzazioni e dei servizi, ma sono considerati spazi di campo i quartieri e l'università stessa. Nella fase di campo gli studenti sono accompagnati da un tutor con la funzione di facilitatore rispetto alle funzioni organizzative e l'inserimento in comunità.

La partecipazione ad un corso sperimentale, di durata variabile, che non riconosce crediti agli 
studenti non di medicina, deve essere considerato come bias di selezione, dal momento che seleziona studenti già sensibilizzati ai temi in oggetto. Gli studenti che hanno partecipato alle due edizioni provengono principalmente dal Corso di Laurea in Medicina, Infermieristica, Antropologia, Servizi Sociali, Psicologia, Lettere, specializzandi di Igiene e Medicina Preventiva, e giovani medici.

Per quanto riguarda le metodologie di raccolta dati durante il corso, ogni lezione è valutata attraverso due prospettive: una quantitativa (la classica valutazione di gradimento su scala di 3 , la lezione mi è piaciuta, indifferente, non mi è piaciuta) e una che potremmo definire qualitativa (secondo il classico schema che cosa ti è piaciuto, che cosa non ti è piaciuto, suggerimenti). Le valutazioni vengono raccolte in un unico foglietto distribuito ad ogni studente all'inizio della lezione e restituito alla fine. Durante le lezioni i tutor redigono un diario di campo collettivo in cui vengono annotate le impressioni rispetto al dibattito in aula ed ai temi emersi. Durante la fase di campo, invece, sono gli studenti stessi a dover redigere un diario di campo collettivo, per ogni sottogruppo di esperienza. Alla fine delle attività i diari sono analizzati includendo studenti "volontari": dal momento che l'attività di analisi dei materiali del corso rappresenta un carico orario rilevante, non può essere considerata una parte "obbligatoria". Alla fine del processi di analisi delle valutazioni e del contenuto dei diari di campo, ${ }^{\mathrm{ix}}$ viene effettuato un focus group per discutere ed interpretare con gli studenti che si sono resi disponibili i risultati ottenuti. In questo articolo ci riferiremo ai temi emersi rispetto al lavoro ed alla formazione interprofessionali.

\section{RISULTATI}

L'analisi delle valutazioni quantitative del corso hanno riportato un giudizio neutro o positivo nel $100 \%$ dei casi. Bisogna ribadire però il bias di selezione degli studenti, che gioca un ruolo rilevante nella soddisfazione degli stessi. Le valutazioni di tipo qualitativo sono state analizzate insieme ai diari di campo: di seguito sono presentate le categorie che riguardano il tema della formazione interprofessionale.

\section{FORMAZIONE INTERPROFESSIONALE UNA SFIDA PER GLI EDUCATORI}

Dall'analisi delle valutazioni emerge come la durata del corso sia breve rispetto alle tematiche affrontate. Un numero di ore ridotto rappresenta una sfida concettuale per docenti e studenti e problematizza il corso in sé, la 
teoria e le metodologie di insegnamento nel contesto interprofessionale. Una prima difficoltà è quella di sintetizzare i contenuti di varie discipline perché siano chiari, comprensibili ma concisi:

\section{"rispetto all'esposizione dei temi teorici, la mia percezione è stata che il docente e gli studenti di quella area, pensassero che gli altri potessero intendere, come se tutti avessero una base per intendere" (student@ di antropologia).}

Gli studenti incontrano difficoltà concettuali, di lessico e relative alle metodologie didattiche:

\section{"A dire il vero io mi aspettavo lezioni frontali, ma quelle che ho trovato non sono state lezioni classiche. Confesso che all'inizio non mi sono piaciute, mi sono sembrate strane, ma poi ho visto che funzionava" (student@ di antropologia).}

Le impressioni emerse confermano l'idea che la didattica interprofessionale non dovrebbe essere confusa con la semplice giustapposizione di differenti conoscenze provenienti da ambiti disciplinari differenti, ma dovrebbe concentrarsi sugli aspetti specifici di produzione di metaconoscenze e metacompetenze:

"Posso dire che i temi trattati sono innovativi, ci sono cose che non avevo mai sentito durante il mio percorso universitario, ma non è solo il punto di vista teorico che che fa la differenza, perché se si modifica il punto di vista un certo argomento diventa proprio un altra cosa" (medic@).
Le stesse riflessioni valgono per il lavoro interprofessionale in campo:

\section{"studiare l'epidemiologia e vedere}

l'epidemiologia non è la stessa cosa, studiare il contesto che sta fuori dall'ospedale e stare in quel contesto fa tutta la differenza, e farlo in gruppo multiprofessionale lo è ancora di più. Per avere un'idea, puoi discutere con i tuoi colleghi del gruppo, ma quando siamo usciti dall'aula tutto quello che avevamo discusso ha cambiato di senso non in termini di rendere le nostre discussioni più vicine alle persone, di collocare cose astratte nell'ambito della realtà, no non in questo senso. Quello che cambia è proprio la tua modalità di intendere la teoria, che cosa riesci a capire rispetto a quello che tenti di descrivere" (sudent@ di medicina).

In altri termini emerge l'idea che quando si mettono al centro della scena le persone, i loro gruppi familiari o le reti sociali di appartenenza, i determinanti di salute che ne definiscono le esistenze, le relazioni tra il piano locale e quello globale, la formazione interprofessionale è in grado di stimolare un mèta apprendimento che non dipende delle singole discipline coinvolte, ma da come queste sono articolate e focalizzate sulla complessità. Nel passaggio dalle lezioni teoriche ai lavori in gruppo ed in campo il tema emerge chiaramente:

"riteniamo la nostra disciplina importante ed è importante quando si parla di teoria, ma quando si passa dal sapere al saper fare e al saper fare in campo con la comunità o in gruppo, quello che ti insegna l'università passa in secondo piano e gli aspetti organizzativi e interpersonali diventano centrali" (studente@ di antropologia);

"nel lavoro in gruppo ho molto apprezzato la parte in cui dovevamo pensare in come organizzarci, in come organizzare il lavoro, nelle cose che dovevamo fare e come farle.

D'altra parte devo ammettere che non sapevamo che cosa fare" (studente@di medicina). 
Per molti degli studenti le lezioni metodologiche, rispetto al lavoro in gruppo e le modalità di relazione in un setting comunitario, sono una novità, mostrando come queste non siano parte del percorso di formazione professionale:

"nella parte teorica parlavamo degli strumenti di lavoro in gruppo, ma non è facile. lo penso che questa dovrebbe essere la prima lezione del corso e che da subito dovremmo essere stimolati ad auto organizzare il lavoro in gruppo magari definendo il ruolo di ciascuno... non so può essere che questo avrebbe potuto aiutarci a cominciare in un modo più rapido perchè è stato difficile. D'altra parte è anche vero che in questo modo ho appreso molte cose per esempio ad avere iniziativa" (student@diantropologia).

Le skills relazionali ed organizzative, dunque, vengono rapidamente considerate come centrali nel lavoro fuori dall'aula, ma data la mancanza di formazione specifica possono essere confuse con capacità individuali, legate alla personalità dei singoli:

"a dire il vero, il differente sguardo intersettoriale è emerso più durante la formazione in aula che durante il lavoro in piccoli gruppo o in campo. Nei gruppi o in campo quello che emerge di più è la visione personale ed in aula ci si presenta più con la propria dimensione disciplinare" (student@ di medicina);

"quello che mi ha lasciato più sorpreso è che la capacità di articolare la teoria con la realtà dipende molto dalla tua personalità, alle volte dai tuoi valori, voglio dire... da quanto ti importa di articolarli" (student@ di medicina); "nella interprofessionalità ti devi confrontare con persone che non solo non intendono la tua posizione, ma addirittura il perchè tu abbia quella posizione (risata), ma il lavoro interprofessionale è possibile se c'è una relazione umana, se puoi valorizzare l'altro, se tu puoi avere fiducia nell'altro." (specializzand@).

Nelle impressioni degli studenti lo sviluppo di metaconoscenze e metacompetenze necessita di un cambiamento culturale che coinvolga I'Università ancor prima che i Corsi di Laurea ma questo rappresenta adesso un limite:

\section{"Probabilmente l'intersettorialità è qualcosa}

che come infermieri pratichiamo di più a differenza di altri Corsi di Laurea, ma alla fine seguiamo diverse materie tutte separate, qui mi pare che stiamo discutendo un'altra cosa: avere la possibilità di confrontarsi col punto di vista degli altri e non ascoltare docenti di altre aree uno alla volta. A mio parere questo è molto importante, e mi chiedo, oggi qui ci sono studenti di varie facoltà lavorando insieme e apprezzando il lavoro interprofessionale, ma se ci fossero anche i docenti lo apprezzerebbero lo stesso?" (student@di infermieristica);

"se la finalità è esplorare la multi-disciplinarità e poi tornare nel proprio contesto di formazione è una cosa, ma se al contrario, la finalità è creare cose differenti allora bisogna organizzarle in modo differente" (student@ di medicina);

"al di là di tutto, nell'università, ci sono già molte proposte formative che potrebbero essere messe in rete, ma che non lo sono. Non so se qualcuno nell'università ha idea che già si sta offrendo qualcosa che potrebbe servire come base per costruire una rete. L'Università in se soffre per una mancanza di integrazione $e$ dialogo interno" (student@ di antropologia); "allo stesso tempo, mi pare molto chiaro, come la trasformazione dovrebbe essere più radicale. 
Una educazione intersettoriale non può essere di una sola facoltà: sarebbe una contraddizione pensare in un corso interdisciplinare e non costruire un percorso interdisciplinare nella Facoltà" (student@di antropologia).

Si può evincere dunque, che così come evidenziato dalla letteratura, è necessario costruire percorsi e contenitori di approssimazione interprofessionali, in cui anche tutor e docenti possano, a partire da questioni epidemiologiche o cliniche concrete, sviluppare contenuti e metodologie di apprendimento centrate sulle metaconoscenze e metacompetenze; la necessità di strutturare percorsi di formazione e ricerca interdisciplinare che consentano ai docenti di approssimare i quadri teorici e metodologici, e fornire agli studenti setting di formazione coerente con il carico di conoscenze e competenze necessarie a confrontarsi con la complessità.

I corsi brevi e opzionali, dunque, possono essere considerati come tappa di un percorso più ampio, un momento di riflessione iniziale in cui mettere in discussione la capacità di problematizzare alcune certezze e portare gli studenti fuori dalla zona di comfort e dove le istituzioni di insegnamento possano sperimentare quadri teorici, ${ }^{\mathrm{x}}$ metodologie e strategie didattiche come evidenziato dai seguenti commenti:

"è qui che sta il problema, si deve riflettere rispetto a dove e come poter utilizzare le conoscenze che abbiamo acquisito durante il corso prima che queste si perdano" (student@ di infermieristica);

" ma se continuiamo a stare nella stessa struttura, dove lo studio è diretto a superare esami, etc etc, questi obiettivi si perderanno rapidamente" (student@ di medicina);

ORGANIZZAZIONE UNIVERSITARIA ED EFFETTI SULLA FORMAZIONE INTERPROFESSIONALE

Oltre a sottolineare l'importanza di un cambiamento culturale nell'impostazione curricolare, dalla nostra ricerca è possibile analizzare gli effetti dell’attuale organizzazione curriculare sulla capacità di lavoro interprofessionale. Nell'interpretazione del materiali raccolti abbiamo voluto usare la categoria del hidden curriculum, ${ }^{x i}$ ossia il processo pedagogico invisibile ed implicito inscritto nell'organizzazione curriculare e nelle pratiche formative. In particolare ci siamo soffermati sull'analisi degli effetti del curriculum invisibile sulla capacità degli studenti di gestire le risorse, pianificare e negoziare nel lavoro in campo:

di sicuro fa la differenza mettere insieme persone che non si conoscono ma che stanno lì 
per mettersi in gioco oppure no. Fa differenza se nel lavoro in gruppo qualcuno di propone e dice "so fare questo ed è questo che posso collocare a disposizione» o se nel gruppo ci sono solo persone che stanno sulla loro"

(student@di lettere);

"se cambia la composizione del gruppo, se c'è qualcuno che segue il corso principalmente per ottenere i crediti,[...] credo che per ciascuno di noi il corso ha un obiettivo differente, e questo

influenza le dinamiche. Per esempio, nel prendere appuntamento per le attività auto organizzate, può essere un caso, ma chi ottiene i crediti dal corso, paradossalmente è chi ha più problemi di agenda e meno flessibilità" (student@di antropologia).

Emergono da questo tipo di commenti due diverse questioni tra loro fortemente correlate: da un lato la mancanza di capacità nella gestione del tempo; dall'altro la rigidità dei percorsi di formazione. Nel commento precedente, per esempio, si fa riferimento alla difficoltà di organizzare le attività proprio per gli studenti che ricevono i crediti, quelli di medicina. Medicina e Infermieristica sono tra i Corsi di Laurea che meno lasciano spazio all'auto-organizzazione degli studenti, sia in termini di tempo che di flessibilità curricolare. $E^{\prime}$ interessante notare che gli studenti di area non sanitaria, quelli non gravati dalla burocrazia del riconoscimento internazionale del titolo professionale, possiedono una maggiore autonomia nella gestione dei loro piani formativi, con una presenza consistente di esami e tirocini a scelta che devono essere pianificati di anno in anno ma che vedono protagonista lo studente stesso. L'altro elemento interessante si riferisce agli studenti che "partecipano al corso solo per i crediti", questione ricorrente riferita non sono al corso elettivo ma esteso a tutto il percorso universitario

"ma se continuiamo a stare nella stessa struttura, dove lo studio è diretto a superare esami, etc etc, questi obiettivi si perderanno rapidamente".

Possiamo avanzare l'idea che la mancanza di autonomia degli studenti dei Corsi di Laurea più normativi sia solo apparente ma, in analogia con quanto riportato nella letteratura di analisi istituzionale, ${ }^{\text {xii }}$ nelle organizzazioni si possono evidenziare le strategie con cui $\mathrm{i}$ soggetti coinvolti riorganizzano nello spazio micropolitico la loro autonomia in termini di tempo e spazi. La cosa interessante nel nostro caso è comprendere quali siano gli effetti di queste strategie sulla formazione dei sanitari, dal momento che proprio per la loro natura di riorganizzazione micropolitica sono fuori dal campo delle valutazioni istituzionali, possono contravvenire alle regole stabilite dall'organizzazione e per questo essere deliberatamente occultate. II "seguire il corso solo per i crediti" fa emergere un sistema di 
doppia morale in cui da un lato le necessità burocratiche costituiscono un curriculum obbligatorio ma non pensato a misura di studente (se non a misura di uno studente ideale o medio), dall'altro si comprende come il rispetto dell'organizzazione standardizzata non è la modalità di apprendimento che garantisca allo studente soggetto la migliore formazione. Le pratiche micropolitiche che gli studenti mettono in atto, quando quester contravvengono alla burocrazia sono nascoste o omesse al docente (che probabilmente le reprimerebbero in analogia con la teoria del "consumatore difettoso" di Bauman) e questo ha effetto sulla negoziazione degli obiettivi didattici e sulla gestione all'interno dei gruppi di

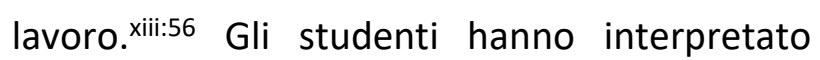
questo processo attraverso tre differenti prospettive: hanno riconosciuto l'esistenza di fattori da loro definiti come "strutturali prossimi", come il tempo e lo spazio fisico per incontrarsi, quali limiti all'organizzazione flessibile delle attività; hanno valutato il conflitto tra agli obiettivi dichiarati del corso e gli obiettivi individuali dello studente come una causa di retrazione dalla possibilità di negoziazione concreta delle attività in gruppo; ed lo hanno indicato come causa disempowerment ed impedimento allo lo sviluppo delle abilità organizzative:

"con il lavoro di quest'anno e dell'anno scorso (edizione 2016 N.d.R.) è successa la stessa cosa, siamo stati molto contenti di avere più autonomia, ma non sapevamo come usarla" (student@ di atropologia).

"per me è stata molto importante la lezione che abbiamo fatto a metà corso sull'organizzazione altrimenti non avrei saputo superare i problemi nell'organizzazione dei gruppi" (specializzand@);

"nel gruppo non tutti hanno partecipato allo stesso modo, abbiamo avuto difficoltà ad organizzare gli incontri, ed abbiamo dovuto sviluppare la maggior parte del lavoro in maniera virtuale, non c'è stata una vera cooperazione perché qualcuno non era mai disponibile"(student@ di medicina);

"non stiamo insieme per sola burocrazia c'è anche una modalità di stare insieme conviviale, ci sono piccole cose che possono essere fatte che consentono di costruire relazioni migliori" (student@di medicina).

Le attività didattiche richiamano ad una dimensione interprofessionale in cui devono essere considerati i macro fattori (quelli che gli studenti hanno definito "fattori strutturali distali") che rimettono alle stratificazioni sociali, differenti traiettorie di vita (e relazioni di potere) che esistono tra le professioni e la necessità di pensare obiettivi didattici diretti a problematizzarle e collocare in scena tutti 
questi fattori che definiscono il quotidiano di lavoro dei professionisti.

La domanda finale di una studentessa è:

è possibile acquisire competenze per lavorare in autonomia e in gruppi intersettoriali attraverso un curriculum così strutturato e normativo? Ma dunque, prima di poter formare professionisti con questa capacità e attitudine dobbiamo prima cambiare le università?"(medic@)

\section{CONCLUSIONI}

La ricerca, seppure esplorativa e realizzata su un gruppo di studenti selezionati, sembra confermare il ruolo positivo della formazione interprofessionale, arricchita dalla componente di lavoro in comunità, rispetto al confronto con la complessità che il campo della salute attualmente presenta. Inoltre, l'alleanza tra Università e comunità risponde alla terza missione dell'Università stessa e potrebbe pertanto essere un ulteriore stimolo alla priorizzazione di percorsi di formazione interprofessionale. In accordo con quanto detto la ricerca suggerisce la necessità dello sviluppo di specifici percorsi di formazione interprofessionali curriculari, che consentano a docenti e studenti di sviluppare in maniera adeguata le metaconoscenze e le metacompetenze necessarie. Questi percorsi dovrebbero essere strutturati direttamente a livello di Ateneo, in modo da garantire lo spazio istituzionale necessario e facilitare il superamento della frammentazione disciplinare. D’altra parte la formazione interprofessionale deve fare i conti con fattori strutturali, alcuni dipendenti dalle condizioni locali in cui la formazione avviene (per usare il gergo degli studenti i fattori "strutturali prossimi"), altri che implicano forme dell'organizzazione sociale che non sono prossime agli scenari di formazione-intervento. In tutti i casi questi fattori agiscono come determinanti, influenzando in maniera estensiva i processi pedagogici, e devono essere attentamente studiati e corretti. La metodologia di ricerca-formazione-intervento si è dimostrata adeguata alla comprensione dei processi pedagogici e nel fornire indicazioni sulla possibile organizzazione delle attività di formazione e si candida, quindi, ad essere utilizzata come metodologia attiva di gestione e valutazione dei processi pedagogici in scenari complessi. 


\section{REFERÊNCIAS}

${ }^{1}$ WHO. Transforming and scaling up health professionals' education and training: WHO Education Guidelines 2013. Geneva: WHO; 2013

${ }^{2}$ Fals-Borda O, Anisur-Rahman M, editors . Action and knowledge: breaking the monopoly with participatory action research. New York: Apex Press; 1991.

${ }^{3}$ Martino A, Marta BL, Sintoni F, et al. Pratiche di Ricerca-Formazione-Intervento nel contesto italiano. In: Franco T, Ceccim R, editors. Prassi in Salute Globale. Porto Alegre, Bologna: Rede UNIDA/CSI-Unibo; 2016

${ }^{4}$ Freire P. Pedagogia do oprimido. 12.ed. Rio de Janeiro: Paz e Terra; 1983.

${ }^{5}$ Freire P. Pedagogia da autonomia: saberes necessários à prática educativa. São Paulo: Paz e Terra; 2011.

${ }^{6}$ Heller A. Every day life. London: Routledge; 1984

${ }^{7}$ Bloom B S, editor. Taxonomy of Educational Objectives: the classification of educational goals. David McKay Company; 1956.

${ }^{8}$ Mclaren P, Kincheleoe JL. Rethinking critical theory and qualitative research. In: Zou Y, Trueba ET, editor. Ethnography and schools: qualitative approaches to the study of education. Lanham: Rowman and Littlefield; 2002. p. 87-138.

${ }^{9}$ Downe-Wamboldt B. Content analysis: method, applications, and issues. Health care for women international. 1992; 13(3):313-321.

${ }^{10}$ Brown M. Comfort zone: Model or metaphor? Journal Outdoor Environmental Education. 2008; 12(1):3.

${ }^{11}$ Hafferty F. Beyond curriculum reform: confronting medicine's hidden curriculum. Acad Med. 1998; 73(4):403-407.

${ }^{12}$ Franco TB, Merhy EE. Cartografias do Trabalho e Cuidado em Saúde. Tempus Actas Saúde Coletiva. 2012; 6(2):151-163.

${ }^{13}$ Bauman Z. Consuming life. Cambridge; Polity Press; 2007.

Figura 1 Ricerca-Formazione-Intervento, Martino et al. 2014

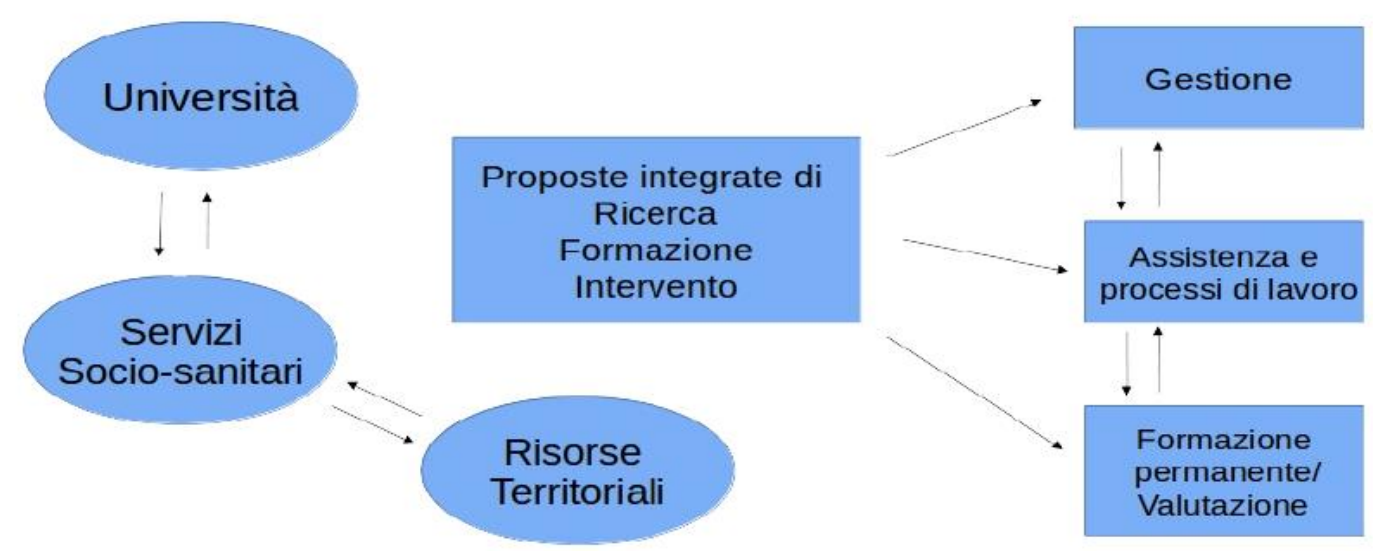

